\title{
Occupational asthma caused by a plastics blowing agent, azodicarbonamide
}

\author{
AJM SLOVAK \\ From Fisons Occupational Health Service, Loughborough, Leicestershire
}

\begin{abstract}
A prevalence study of occupational asthma was carried out by questionnaire in 1980 among a group of 151 workers who had been or were exposed to azodicarbonamide dust in the process of its manufacture. Twenty-eight $(18.5 \%)$ people without previous asthma gave a history of episodes of late onset asthma after exposure to azodicarbonamide. Re-exposure caused repetition and worsening of symptoms. Immediate removal from further exposure resulted in rapid cessation of symptoms without further recurrence. Seven of 13 sensitised individuals who were still exposed three months after the onset of disease developed prolonged airways hyperreactivity to common environmental irritants. Azodicarbonamide should be excluded as a causative agent in plastics and rubber industry workers complaining of occupational asthma.
\end{abstract}

Azodicarbonamide (AC) is a condensation product of urea and hydrazine and is widely used as a blowing agent in the manufacture of expanded foam plastics. It is manufactured predominantly as a fine yellow powder and is milled to particle sizes in the 2-10 micron range. This technically most useful particle size distribution is almost exclusively in the respirable range. It may be mixed with other chemicals to modify performance. The main volume usages are in the manufacture of polyvinylchloride and polyolefin plastics for wallcoverings, floor coverings, insulation and packaging materials.

During the manufacturing history of azodicarbonamide a number of cases of occupational asthma have come to light. Two cases have been reported among workmen in the USA. ${ }^{1}$ This study surveys the prevalence of cases of asthma in a group of work people engaged in $\mathrm{AC}$ manufacture and describes the clinical characteristics and relationship with atopic status.

\section{Methods}

All 151 personnel still in works employment who had been or were currently engaged in manufacture, servicing and quality control on the AC plant since its opening in 1966 were included in the survey. Preliminary screening was carried out by an objective, nurse-administered questionnaire, followed by a structured, clinical, occupational history taken by

Address for reprint requests: Fisons Pharmaceutical Division, 12 Derby Road Loughborough, Leics. the author from which a diagnosis was made. The diagnosis of asthma was regarded as positive on a history of repeated episodes of wheezing or chest tightness ( \pm cough) related to exposure to AC. In 12 out of 28 cases it was possible to confirm this from medical notes made at the time of attacks. Twelve further cases could be identified from the notes of people who had left company employment but because the AC plant acted as the reception and training plant for most new entrants into the works, between-plant comparison of rates of manpower wastage offered no valid indication of possible illhealth associated with covert AC sensitisation.

Pre- and post-shift spirometry was performed on three groups of people from within the total population. These were (a) persons diagnosed as having had asthma caused by AC, (b) all asymptomatic process workers in daily exposure who had been on the plant for more than one year, and (c) a control process worker population without any contact with the product or other lung sensitisers. Spirometry was performed with a wedge-bellows spirometer (Vitalograph) and $F E V_{1}, F V C$, and $F E V_{1} / F V C$ ratio were recorded. A small group of 11 personnel, from among those who were diagnosed as having had asthma caused by AC, were issued with Wright minipeak flow meters to obtain evidence of the efficacy of measures taken to protect or remove sensitised personnel from exposure. Peak flow measurements were self-recorded two-hourly during waking hours for a three-month period outside the hay fever season. 
Azodicarbonamide is insoluble in all common solvents and considerable difficulty was experienced in finding a safe solvent for prick testing. Eventually $0.1 \%, 1 \%$, and $5 \%$ solutions of AC in dimethylsulphoxide were used. Prick tests were also performed to determine atopic status. Positive status was defined by a $3 \mathrm{~mm}$ wheal to any one of the following: cat, house dust, grass mixture, or aspergillus.

For this survey, dust levels on the manufacturing plant were measured using standard techniques of industrial hygiene with personal sampling by Casella pump followed by gravimetric analysis of samples.

The use of challenge studies in the occupational setting was not considered to be justified.

\section{Results}

\section{DUST LEVELS}

Azodicarbonamide is a chemical of very low acute toxicity and the plant was therefore not designed to contain the product totally. This was reflected in quite high time weighted average levels in the range $2-5 \mathrm{mg} / \mathrm{m}^{3}$. Historical levels are not available but are likely to have been similar, the plant having remained largely unchanged since opening.

\section{CLINICAL CHARACTERISTICS}

The prevalence of workers diagnosed as having developed asthma because of AC was $18.5 \%$ (28). None of these men had had asthma or any other significant chest disease before exposure to the chemical.

Table Age and smoking habits of cases and sample population

\begin{tabular}{lll}
\hline Age & Cases & Sample \\
\hline Range & $29-63 \mathrm{yr}$ & $17-64 \mathrm{yr}$ \\
Mean & $41 \mathrm{yr}$ & $40 \mathrm{yr}$ \\
Smoking status & $25 \%$ & $29 \%$ \\
Life-time non-smoker & $32 \%$ & $29 \%$ \\
Ex-smoker $(1 \mathrm{yr}+)$ & $43 \%$ & $42 \%$ \\
Smoker & $100 \%$ & $100 \%$ \\
Totals & & \\
\hline
\end{tabular}

Over half the patients developed asthma within three months of first exposure and $75 \%$ developed it within the first year. The predominant symptoms in all cases were shortness of breath, chest tightness, and wheezing. Additional symptoms complained of were cough $(39 \%)$, rhinitis $(29 \%)$, conjunctivitis $(25 \%)$, and rash $(4 \%)$. The three different patterns of onset and duration of asthma, described by patients, conform to the immediate, late, and dual patterns reviewed by Pickering $;{ }^{2} 56 \%$ had late onset asthma, $22 \%$ immediate, and $22 \%$ dual. All persons with a dual response pattern, except one, had it develop upon continued patient exposure from what had originally been a late pattern attack. However, it should not be inferred that these patterns of asthma would be directly reproduced on challenge testing. Altogether $46 \%$ of patients reported noticeable worsening of their condition with continuing exposure, perceived both as worsening of symptoms and shortening of time between going on the plant and the reappearance of symptoms.

Forty-four per cent of patients had reported their condition to the occupational health department and had been quickly transferred to other work. Fiftysix per cent had not reported, but it is interesting to note that all had eventually recognised the cause of their symptoms and had made their own arrangements to transfer or keep away from working with AC. Thirteen $(46 \%)$ remained in working exposure for more than three months after developing asthma; over half of these developed subjective airways hyperreactivity which persisted more than one month after removal from exposure. No such persistence was noted in personnel removed from exposure less than three months from first symptoms. Subjective airways hyperreactivity evinced by previously tolerated general irritants-for example, sulphur dioxide, tobacco smoke-persisted in five of eight men for over three years after removal from exposure; two of these still have exercise-induced asthma after seven years although this too has improved as judged by decreased need for prophylactic and symptomatic treatment.

\section{LUNG FUNCTION TESTS}

Pre- and post-shift spirometry in the groups $a, b$, and c studied revealed nothing but the small improvement in daytime performance to be expected from diurnal variations. There was no evidence of reversible airflow obstruction among those seen and no attacks were observed during this study.

Peak flow measurements in sensitised personnel transferred away from exposure also showed no variation between patterns recorded during holiday periods and at work. This suggests that they do not now have any significant exposure to AC.

\section{A TOPY}

It appears that atopics were not selected out by pre-employment examination. Identical proportions (48\%) were found among AC sensitised personnel and unsensitised asymptomatic AC-exposed process men of more than one year's on-plant service. It is unlikely therefore that atopy is predictive of predisposition to AC-sensitivity.

PRICK TESTS

Prick tests to all the concentrations of $\mathrm{AC}$ solutions 
used $(0 \cdot 1,1$, and $5 \%)$ were negative in AC-sensitised and asymptomatic exposed workers alike.

\section{OTHER VARIABLES}

A low background level of chest disease was noted in the population under study. No pre-exposure cases of asthma were noted: four chronic bronchitics were found. This reflects both self-selection among job candidates and exclusion of those with overt chest disease at pre-employment medicals. The impact of age distribution and smoking habits on the data was considered and not found significant (see table).

\section{Discussion}

At least three mechanisms have been cited as contributing to the causation of occupational asthma. These are immunological, para-immunological, and non-immunological. ${ }^{2}$ Further, it is useful to separate non-immunological mechanisms into non-specific inflammatory response (irritancy) and specific pharmacological activity, as is postulated for toluene diisocyanate. ${ }^{3}$ In the case of AC, the characteristic clinical presentation consisted of a latent period before onset, followed by abrupt onset and frequent rapid worsening of symptoms if exposure continued. Asymptomatic workers, exposed for over a year to the relatively high dust levels on the plant, showed no diminution on comparison of pre- and post-shift spirometry. It is probable therefore that $\mathrm{AC}$ has no irritant or pharmacological bronchoconstrictive effect and therefore non-immunological causative mechanisms can be excluded in asthma caused by AC. Only two of the 28 sensitised workers ever received sodium cromoglycate and both reported alleviation of symptoms.

The results presented suggest that $\mathrm{AC}$ can be viewed as a potent lung sensitiser of the small molecular weight type, the characteristics of this type being a predominance of severe and worsening late onset asthmatic symptoms usually occurring within the first year of exposure and associated with negative skin prick tests to the causative agent. This may be contrasted with the disease caused by high molecular weight sensitisers such as the antigens of laboratory animal allergy. Here the predominant symptom in prevalence studies is rhinitis without asthma. Only a minority get asthma, which develops from rhinitis after one to two years on average and is associated with positive skin prick tests. The symptoms may never be very severe. ${ }^{4}$

Among those who developed AC-induced asthma was a group of $13(46 \%)$ whose prolonged exposure to AC resulted in airways hyperreactivity which continued more than a month after cessation of exposure to that substance. In all cases subjective well being has gradually improved but this has sometimes taken several years. It is worth recording that this slow recovery from bronchial hyperreactivity does not exclude a good prognosis.

There was no difference in the prevalence of atopy in AC-sensitised and non-sensitised AC exposed workers. Nor was there any evidence of pre-employment or work-induced discrimination against atopics. Indeed there was a slight excess of atopics in both groups when compared with the general population. 5

It appears that atopic status is not predictive of AC sensitisation. Negative correlations of sensitisation to atopy have also been noted in the pharmaceutical industry 6 and in the electronics industry. ${ }^{7}$ It seems timely to reconsider the value of the widespread occupational medical practice of automatically excluding atopics from work with lung sensitisers.

The work of Burge 8 on serial peak expiratory flow rate (PEFR) measurements in the workplace suggests that caution must be exercised in the conclusions drawn in sensitised workers from pre- and post-shift spirometric data. The pre-shift reading cannot be taken as a true unaffected baseline since the airways may still be maximally constricted from previous exposure. Similarly the absence of a drop over a shift cannot be taken as evidence of absence of an effect. However in asymptomatic exposed workers it was possible to use spirometry to demonstrate the absence of any subclinical broncho-constrictive effect of AC. Measurement of serial PEFR in this study was confined to a group of AC sensitised workers to test the hypothesis that they had been effectively transferred away from exposure to AC and this was confirmed.

Thirty-eight per cent of all sensitised workpeople had sought advice from their own doctors during periods of illness caused by AC. Understandably, in view of the accompanying symptoms of rhinitis, conjunctivitis, and cough, in some cases, the initial diagnoses made were coryza, influenza, or bronchitis. After repeated attacks of asthma $12 \%$ were identified for what they were. It is interesting that, despite the wide range of known environmental allergens, there is still marked professional reluctance to diagnose asthma and to consider an extrinsic cause in the workplace.

It is estimated that annually, 8000-10000 people in the plastics and rubber industry in the United Kingdom will come into contact with AC during the course of their work. If such workers develop occupational asthma AC should be excluded as the causative agent although toluene diisocyanate (TDI) undoubtedly still remains by far the more frequent sensitiser. Sensitisation in the workplace 
may be avoided by the use of good ventilatory practice and the issue of appropriate protective masks to workers. Dust should be removed from contaminated clothing and from the workplace by vacuuming rather than sweeping and brushing. Contaminated clothing should not be worn outside the workplace.

Further study of AC sensitisation is necessary to characterise the immunological mechanisms involved. This will be done prospectively as new cases arise.

I would like to thank Sister P Woods and Miss DG Podevyn for their clinical and clerical work and Dr RN Hill and Dr JL Bonsall for their very helpful criticism.

\section{References}

${ }^{1}$ Ferris BG, Peters JM, Burgess WA, Cherry RV. Apparent effect of an azodicarbonamide on the lung: a preliminary report. J Occup Med 1977;19:424-5.

2 Pickering CAC. A 7 year survey of "allergic" occupational lung disease in the United Kingdom. Eur J Respir Dis Suppl 1980;107:137-49.

${ }^{3}$ Butcher BT, Salvaggio JE, O'Neil CE, Weill H, Garg O. Toluene diisocyanate (TDI) pulmonary disease: immunopharmacologic and mecholyl challenge studies. $J$ Allergy Clin Immunol 1977;59:223.

4 Slovak AJM, Hill RN. Laboratory animal allergy: clinical survey of an exposed population. Brit J Ind Med 1981; 38:38-41.

${ }^{5}$ Barbee RA. Distribution of atopy in a normal population. Ann Intern Med 1976;84:129-33.

- Taylor G, Davies GE, Altounyan REC, et al. Allergic reactions to laboratory animals. Nature 1976;260-80.

' Burge PS, Perks W, O'Brien M, Hawkins R, Green M. Occupational asthma in an electronics factory. Thorax 1979;34:13-18.

${ }^{8}$ Burge PS. Prolonged and frequent recording of peak expiratory flow rate in workers with suspected occupational asthma due to colophony or isocyanate fumes. MSc in Occupational Medicine 1978, University of London. 